\title{
Molecular Basis of Tumor Heterogeneity in Endometrial Carcinosarcoma
}

\author{
Susanna Leskela ${ }^{1,2, *(\mathbb{D})}$, Belen Pérez-Mies ${ }^{2,3}$, Juan Manuel Rosa-Rosa ${ }^{1,2}$, Eva Cristobal ${ }^{1}$, \\ Michele Biscuola ${ }^{2,4,5}$, María L. Palacios-Berraquero ${ }^{6}$, SuFey Ong ${ }^{7}$, \\ Xavier Matias-Guiu Guia $2,8,9,10$ and José Palacios $1,2,11, *$ (D) \\ 1 Department of Pathology, Institute Ramón y Cajal for Health Research, 28034 Madrid, Spain \\ CIBER-ONC, Instituto de Salud Carlos III, 28029 Madrid, Spain \\ Department of Pathology, Hospital Ramón y Cajal, 28034 Madrid, Spain \\ Department of Pathology, Instituto de Biomedicina de Sevilla (IBiS), 41013 Seville, Spain \\ Hospital Universitario Virgen del Rocío/CSIC/Universidad de Sevilla, 41013 Seville, Spain \\ Department of Hematology and Hemotherapy, Clínica Universidad de Navarra, 31008 Pamplona, Spain \\ NanoString Technologies, Inc, Seattle, WA 98109, USA \\ Department of Pathology, Hospital U Arnau de Vilanova, 25198 Lleida, Spain \\ Department of Pathology, Hospital U de Bellvitge, L'Hospitalet de Llobregat, 08907 Barcelona, Spain \\ 10 IRBLLEIDA, IDIBELL, University of Lleida, 25003 Lleida, Spain \\ 11 Faculty of Medicine, University of Alcalá de Henares, Alcalá de Henares, 28801 Madrid, Spain \\ * Correspondence: susanna.leskela@gmail.com (S.L.); jose.palacios@salud.madrid.org (J.P.); \\ Tel.: +34-91-336-8140 (S.L.); +34-91-336-8337 (J.P.)
}

Received: 24 May 2019; Accepted: 2 July 2019; Published: 10 July 2019

\begin{abstract}
Endometrial carcinosarcoma (ECS) represents one of the most extreme examples of tumor heterogeneity among human cancers. ECS is a clinically aggressive, high-grade, metaplastic carcinoma. At the morphological level, intratumor heterogeneity in ECS is due to an admixture of epithelial (carcinoma) and mesenchymal (sarcoma) components that can include heterologous tissues, such as skeletal muscle, cartilage, or bone. Most ECSs belong to the copy-number high serous-like molecular subtype of endometrial carcinoma, characterized by the TP53 mutation and the frequently accompanied by a large number of gene copy-number alterations, including the amplification of important oncogenes, such as CCNE1 and $c-M Y C$. However, a proportion of cases (20\%) probably represent the progression of tumors initially belonging to the copy-number low endometrioid-like molecular subtype (characterized by mutations in genes such as PTEN, PI3KCA, or ARID1A), after the acquisition of the TP53 mutations. Only a few ECS belong to the microsatellite-unstable hypermutated molecular type and the POLE-mutated, ultramutated molecular type. A common characteristic of all ECSs is the modulation of genes involved in the epithelial to mesenchymal process. Thus, the acquisition of a mesenchymal phenotype is associated with a switch from E- to N-cadherin, the up-regulation of transcriptional repressors of E-cadherin, such as Snail Family Transcriptional Repressor 1 and 2 (SNAI1 and SNAI2), Zinc Finger E-Box Binding Homeobox 1 and 2 (ZEB1 and ZEB2), and the down-regulation, among others, of members of the miR-200 family involved in the maintenance of an epithelial phenotype. Subsequent differentiation to different types of mesenchymal tissues increases tumor heterogeneity and probably modulates clinical behavior and therapy response.
\end{abstract}

Keywords: uterine carcinosarcoma; endometrial carcinoma; metaplastic carcinoma; epithelial-tomesenchymal transition; clonality; mutation; TP53; PI3K/AKT pathway; gene expression; miRNA expression 


\section{Clinicopathological Characteristics}

Endometrial carcinosarcoma (ECS), also known as malignant mixed Müllerian tumor (MMMT), is a high-grade tumor characterized by a biphasic growth of malignant epithelial (carcinomatous) and mesenchymal (sarcomatous) components (Figure 1) [1]. ECS is a rare aggressive neoplasm accounting for approximately $2 \%$ to $5 \%$ of gynecological carcinomas, but it causes around $16 \%$ of all deaths due to malignancies of the uterine corpus [2,3]. Although ECS shares similar risk factors with endometrial carcinoma, such as obesity, nulliparity, smoking, and exogenous estrogen use, they present at more advanced stages and have significantly worse survival than high-grade endometrial carcinomas [3-8].

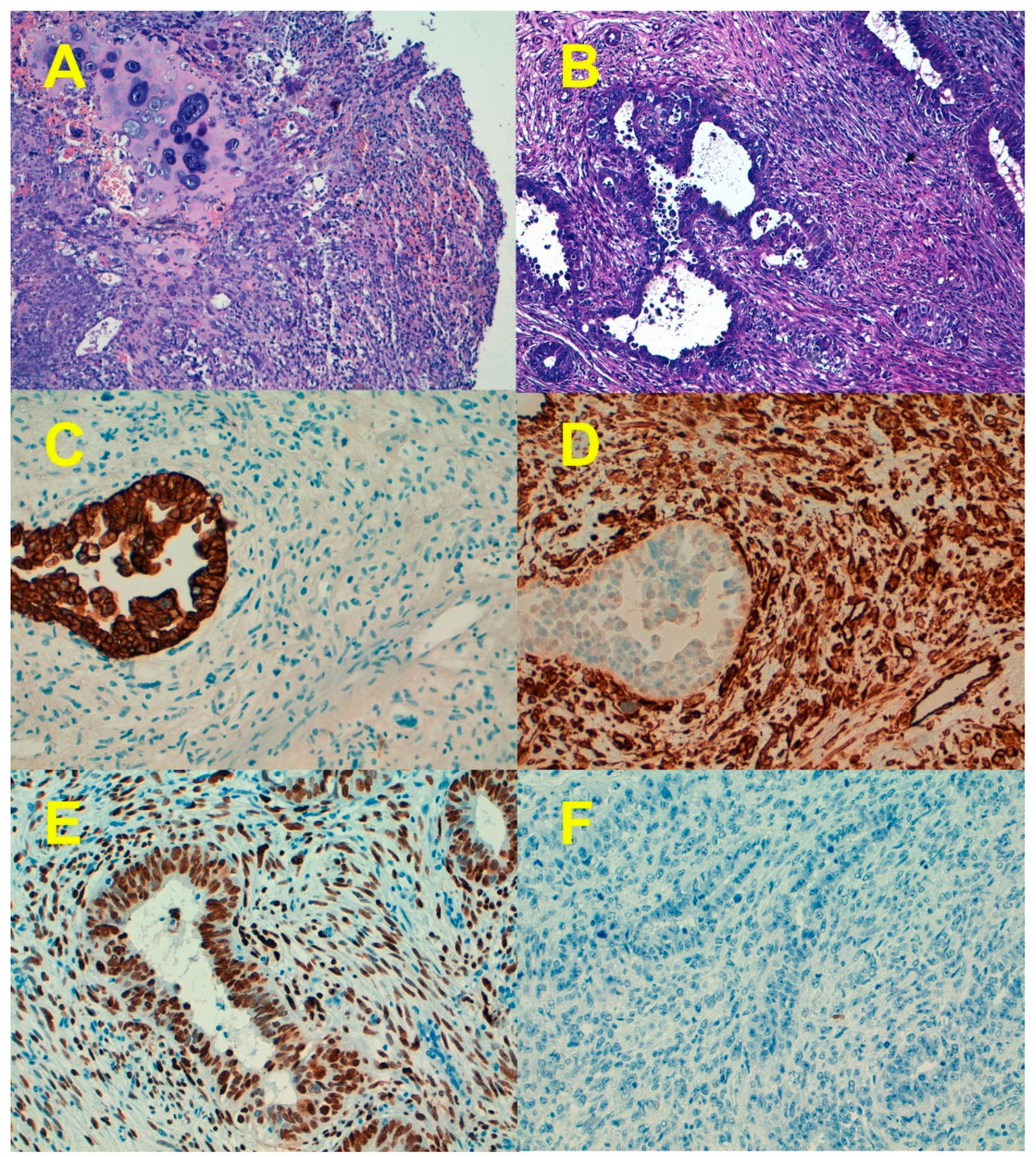

Figure 1. Morphological and immunohistochemical features of endometrial carcinosarcoma. (A) Hematoxylin-eosin staining of an endometrial carcinosarcoma showing the epithelial component surrounded by the heterologous mesenchymal component (chondrosarcoma). (B) Endometrial carcinosarcoma with homologous sarcoma (H\&E). (C) Cytokeratin expression of the case depicted in b. (D) Vimentin expression in the case depicted in b. (E) p53 overexpression in both the carcinomatous and sarcomatous components. (F) p53 null pattern in both the carcinomatous and sarcomatous components. Only occasional normal stromal cells expressed $\mathrm{p} 53$. Original magnification $10 \times$ for $(\mathbf{A}, \mathbf{B})$, and $20 \times$ for $(\mathbf{C}-\mathbf{F})$.

Matsuo et al. [9] analyzed the incidence of ECS in the USA during 1973-2013 in 235,849 primary endometrial carcinomas (ECs) and observed that the proportion of ECS is now significantly higher 
than before and accounts for more than 5\% of ECs. There was a significant rise in the proportion of ECS among primary ECs from $1.7 \%$ to $5.6 \%$ during this period. Moreover, among 76,118 type II ECs, the proportion of ECS also increased significantly from 6\% to 17.5\%; ECS was detected in 11,000 (4.7\%) women. The percentage of black women with ECS was elevated from $11.9 \%$ to $20 \%$, whereas the proportion of white women decreased from $86 \%$ to $60.5 \%$. The possible factors associated with the increase of ECS include the increment in the number of older women and the obese population in the US, and the global increase in the incidence of breast cancer with a concordant increment in tamoxifen use [9].

Several studies have demonstrated that tamoxifen use may be associated with an increased incidence of ECS. In women with breast cancer, the incidence of ECS is 6.35-fold higher in those treated with tamoxifen [10]. Matsuo et al. [11] reported that $\sim 6 \%$ of women with ECS have a history of tamoxifen use and that tamoxifen-related ECS was significantly associated with a higher proportion of stage IA disease (48.4\% versus $29.9 \%)$ and a lower risk of stage IVB disease $(7.8 \%$ versus $16 \%$ ) compared to tamoxifen-unrelated ECS. Deep myometrial tumor invasion was less common in uterine carcinosarcoma related to tamoxifen use (28.3\% versus $48.8 \%)$. However, in spite of these favorable tumor characteristics, tamoxifen-related ECS had comparable stage-specific survival outcomes compared to tamoxifen-unrelated ECS.

From a morphological point of view, the epithelial component of ECS could be endometrioid (most common in most series) or non-endometrioid (serous, clear cell, undifferentiated, or mixed) [3,4,12-15]. Matsuo et al. [16] reported that among 906 ECS evaluated for histological patterns in their series, high-grade carcinoma/homologous sarcoma (40.8\%) was the most common type followed by high-grade carcinoma/heterologous sarcoma (30.9\%), low-grade carcinoma/homologous sarcoma (18\%), and low-grade-carcinoma/heterologous sarcoma (10.3\%). In 75\% to 95\% of ECS, the epithelial component was of high grade $[16,17]$. The mesenchymal component could be minimal or extensive. Sarcoma dominance (SD) is defined by the presence of more than $50 \%$ of the tumor composed by the sarcomatous component. The mesenchymal component could be subdivided into homologous (fibrosarcoma, leiomyosarcoma, and endometrial stromal sarcoma) and heterologous, the latter including skeletal muscle, cartilage, fat, or osteoid, which is present in up to $60 \%$ of tumors $[3,4,12-19]$. Immunohistochemistry may be useful in confirming the presence of a heterologous mesenchymal component, which, as discussed later, is an adverse prognostic indicator in some series. For example, nuclear staining with myogenin and Myoblast determination protein 1 (myoD1) helps to confirm the presence of rhabdomyoblastic differentiation (Figure 2) [20].

Regarding other pathological features, $55 \%$ to $60 \%$ of ECS show less than $50 \%$ of myometrial invasion at diagnosis. Lymphovascular invasion (LVI) prevalence in ECS seems to be higher than in other types of endometrial cancer (60.4-62\% vs. 26-52\%) [21]. Matsuo et al. [21] reported that among LVI-positive cases, LVIs with a carcinomatous component alone was found in 76.8\% and LVI containing a sarcomatous component with or without a carcinomatous component in the remaining $23.2 \%$. Tumors in the LVI-sarcoma group were more likely to have SD (82.1\% vs. $26.4 \%)$, heterologous sarcomatous component (51.3\% vs. 37.9\%), low-grade carcinoma (42.5\% vs. $22.4 \%$ ), and large tumor size $(81 \%$ vs. $70.2 \%)$ in the primary tumor site compared with tumors in the LVI-carcinoma group.

Also, the pattern of metastasis differs between the epithelial and mesenchymal parts of the ECS. Thus, for example, Matsuo et al. [16] analyzed 1096 metastatic sites and showed that carcinoma components tended to spread lymphatically, while sarcoma components tended to spread locoregionally (cervix, vagina, etc.).

ECS follows an aggressive clinical course. Patients with International Federation of Gynecology and Obstetrics (FIGO) stage 1-2 disease have a five-year disease-specific survival of 59\%, while those with stage 3 and 4 disease have a five-year disease-specific survival of $22 \%$ and $9 \%$, respectively [2]. The most important prognostic factors in these tumors include FIGO stage and depth of myometrial invasion [5,7,8,13,15,22]. Other known clinicopathologic features associated with worse outcome are the grade and histology of the epithelial component and lymphovascular invasion [3,5,8,13]. Although 
the grade and the amount of the sarcomatous component and the presence of heterologous elements are not related to the overall outcome in some series $[6,13,18,22]$, recent studies have shown the importance of the sarcomatous component in the prognosis and response to radiotherapy [17,23]. Thus, Matsuo et al. [24] reported that ECS with better prognosis were those composed of a low-grade carcinoma and homologous sarcoma without SD. In contrast, the worse prognosis corresponded to ECS composed of a high-grade carcinoma and heterologous sarcoma and SD. This latter type of tumor tended to occur in older, obese, and Caucasian patients, and they were more likely to have metastatic implants, large tumor sizes, LVI with sarcoma cells, and higher lymph node ratios. Also, SD seems to be a prognostic factor in some series [17,23], and it is associated with loco-regional tumor metastasis and recurrence with sarcoma. In addition, ECS with SD seems more sensitive to radiotherapy compared to ECS without sarcoma dominance [23]. Finally, different studies have reported a poor prognosis in ECS with rhabdomyoblastic differentiation $[4,17,19]$.

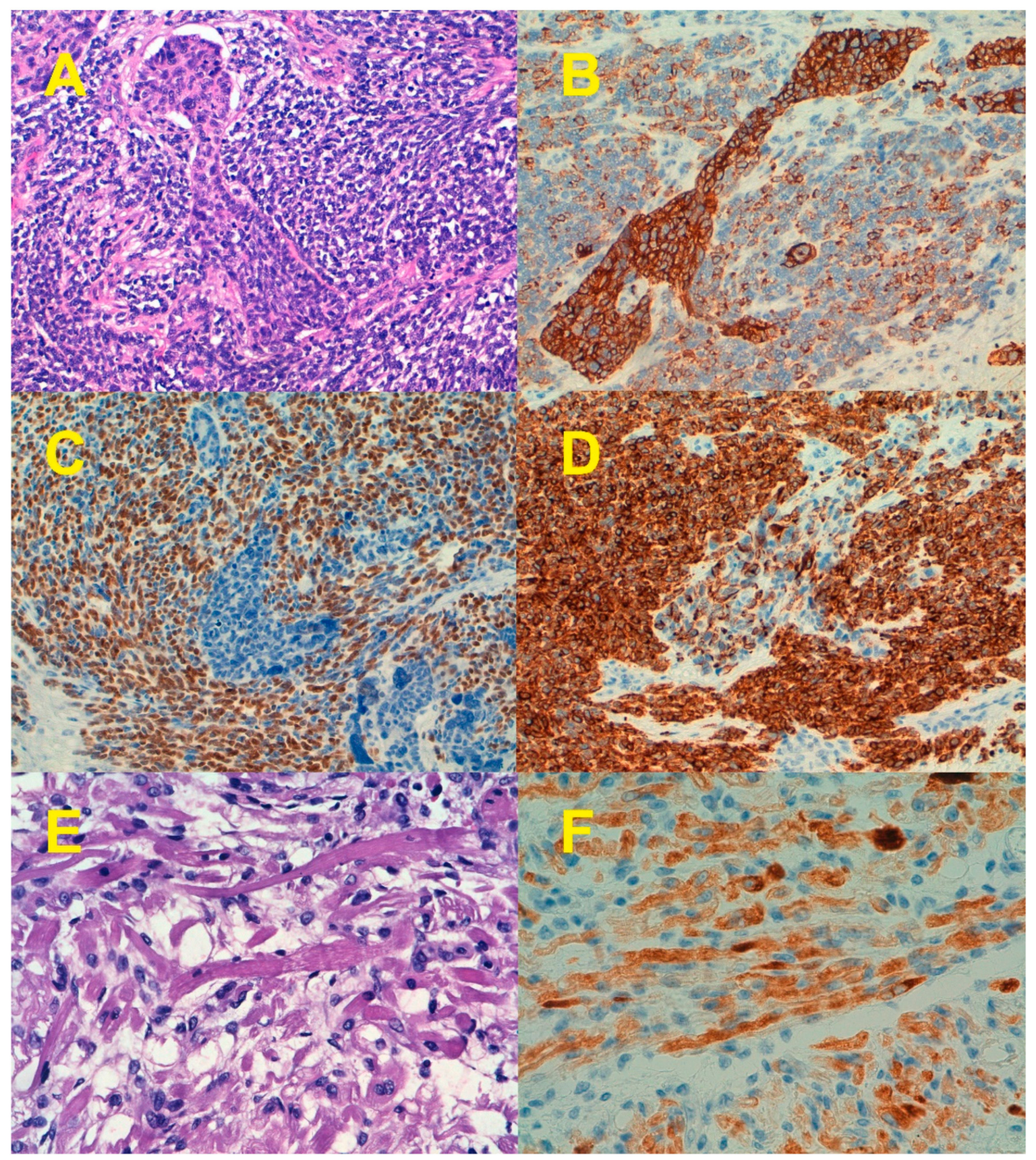

Figure 2. Endometrial carcinosarcoma with rhabdomyoblastic differentiation. Some cells showed an intermediate epithelial/mesenchymal differentiation as suggested by the expression pattern of cytokeratins, myogenin, and desmin. (A) Hematoxylin-eosin staining. (B) Cytokeratin (CK AE1/AE3) expression. (C) Myoblast determination protein 1 (MyoD1) expression. (D) Desmin expression. (E) Striated rhadbomyoblasts (H\&E). (F) Desmin expression by striated rhadbomyoblasts. Original magnification $20 \times$ for $\mathrm{A}-\mathrm{D}$, and $40 \times$ for $\mathrm{E}$ and $\mathrm{F}$. 
Molecular studies have demonstrated similar genetic alterations in both the carcinomatous and sarcomatous components of ECS (Table 1). Thus, it is now accepted that most carcinosarcomas are in fact metaplastic carcinomas, in which the sarcomatous component is derived from the carcinomatous component as a result of transdifferentiation (epithelial-to-mesenchymal transition-EMT) during the evolution of the tumor as shown in several studies $[25,26]$. However, a small percentage of ECS probably represent real collision tumors, since they are molecularly biclonal and most likely develop from two independent cell populations [6,27].

Table 1. Comparison of gene mutation frequency among different histological types of endometrial cancer according to The Cancer Genome Atlas Program (TCGA).

\begin{tabular}{lccc}
\hline \multicolumn{1}{c}{ GENE } & Endometrioid Carcinoma & Serous Carcinoma & Carcinosarcoma \\
\hline PTEN & $82 \%$ & $10 \%$ & $19 \%$ \\
\hline PIK3CA & $54 \%$ & $37 \%$ & $35 \%$ \\
\hline PIK3R1 & $36 \%$ & $11 \%$ & $11 \%$ \\
\hline CTNNB1 & $34 \%$ & $1 \%$ & $2 \%$ \\
\hline ARID1A & $54 \%$ & $8 \%$ & $12 \%$ \\
\hline KRAS & $24 \%$ & $3 \%$ & $12 \%$ \\
\hline CTCF & $31 \%$ & $2 \%$ & $7 \%$ \\
\hline TP53 & $21 \%$ & $88 \%$ & $91 \%$ \\
\hline FBXW7 & $17 \%$ & $24 \%$ & $39 \%$ \\
\hline PPP2R1A & $11 \%$ & $38 \%$ & $28 \%$ \\
\hline CHD4 & $9 \%$ & $18 \%$ & $17 \%$ \\
\hline CCNE1 (ampl.) & $16 \%$ & $26 \%$ & $41 \%$ \\
\hline MYC (ampl.) & $14 \%$ & $24 \%$ & $21 \%$ \\
\hline MECOM (ampl.) & $18 \%$ & $33 \%$ & $18 \%$ \\
\hline PIK3CA (ampl.) & $10 \%$ & $22 \%$ & $11 \%$ \\
\hline ERBB2 (ampl.) & $8 \%$ & $19 \%$ & $9 \%$ \\
\hline
\end{tabular}

\section{Molecular Subtypes of ECS}

Four molecular groups have been defined for ECs: the hypermutated (mismatch repair deficiency), the ultramutated (POLE mutated), the copy-number low, and the copy-number high groups. These groups not only have different molecular alterations but also different prognoses; patients from the ultramutated group show the best prognosis, whereas patients in the copy-number high group have the highest risk of recurrence [28].

Considering the mutational profile (Table 1; see below Section 2. Molecular Subtypes of ECS), most ECSs are similar to serous-like, copy-number high ECs. Thus, in the study by McConechy et al., most tumors had a molecular profile similar to endometrial serous carcinoma (characterized by the presence of TP53, FBXW7, and PPP2R1A mutations and the absence of ARID1A, CTNNB1, KRAS, or $P T E N$ mutations), while part of the tumors displayed an endometrioid carcinoma-like mutation profile characterized by the presence of ARID1A, CTNNB1, KRAS, and PTEN mutations. Based on both combined genetic and immunohistochemical profiles in their cohort, 18 tumors presented serous-like and 11 tumors presented endometrioid-like molecular profiles. There was a good correlation between the histological subtyping (taking into account the morphology of the epithelial component) and the molecular subtyping in 27 of 29 uterine carcinosarcomas (93\%) [29]. More recently Jones et al., applied this classification to their set of tumors, and were able to classify 55 out of 57 tumors, of which $22 \%$ were endometrioid and $78 \%$ serous-like ECS. One sample did not fit in the model due to an 
ultramutated phenotype caused by the POLE mutation, while another had no mutation in the genes used for classification. Interestingly, all 10 stage IV tumors were serous-like [30].

Most of the endometrioid-like ECSs also showed TP53 mutations, implying that TP53 could be involved in the progression of part of the copy-number low endometrioid-like carcinomas to ECSs, as we have previously reported in undifferentiated endometrial carcinoma [31]. Very few ECS belong to the microsatellite-unstable hypermutated molecular type and the POLE-mutated ultramutated molecular type used for the classification of endometrial carcinoma. The molecular heterogeneity present in ECS opens opportunities for targeted therapies.

\section{Serous-Like Molecular Alterations in ECS}

Previous studies combining aberrant expression of p53 and mutational analysis estimated a TP53 mutation prevalence of $50-60 \%[3,12,22,27,32-35]$. However, subsequent studies using Next Generation Sequencing (NGS) techniques have shown that the true frequency of TP53 mutation in ECS is very high, between $64 \%$ and $91 \%$ [29,30,36-40]. In effect, TP53 mutations are the most frequent molecular alterations in ECS (Table 1). The lack of nuclear p53 expression is most commonly detected with indel or nonsense mutations, while missense mutations usually lead to diffuse nuclear p53 immunostaining. Most of the mutations are located in the DNA binding domain, and very few are present in the translocation and tetramerization motifs. In the DNA binding domain, $32 \%$ of mutations are located on known hotspot residues, and the most frequent are the R248Q and R273C/H (12\% and 7\%, respectively) followed by H179R/D, H193R/Y, and S241Y (5\% each), (http://cancergenome.nih.gov/) [41].

The carcinomatous and sarcomatous components show a concordance of $85 \%$ for the p53 protein overexpression and $96 \%$ for the TP53 gene mutation, which points to a monoclonal origin of both components (Figure 1). p16 overexpression (in-block diffuse expression) occurs in about $60 \%$ of ECS simultaneously with TP53 mutations. The concordance of p16 expression between the carcinomatous and sarcomatous components was about $85 \%$ in different series [12,35,42-44]. In addition to TP53, ECSs show mutations in other genes that are also more frequently affected in endometrial serous carcinoma (ESC) than in endometrial endometrioid carcinoma (EEC). Accordingly, mutations of FBXW7 and PPP2R1A have been reported in $19 \%$ to $39 \%$ and $1 \%$ to $38 \%$, respectively, in different series [36-40].

Regarding the BRCA1 and BRCA2 genes, the frequency of ECS in patients carrying germinal $B R C A 1 / 2$ mutations has been analyzed in different studies. The estimated relative risk for mutation carriers is approximately $2 \%$ per year, most importantly among serous carcinoma [45-47]. A recent series has reported that BRCA1/2 were found mutated in $18 \%$ and $27 \%$, respectively of ECS [30], although in the TCGA (The Cancer Genome Atlas Program) series, only BRCA2 mutations were detected and at a lower frequency (5\%) [28]. Carcinosarcoma of the breast and ovary have been reported in some patients with $B R C A 1 / 2$ germline mutations [48-50].

Zhao et al. [51] found an excess of mutations in genes encoding histone $H 2 A$ and $H 2 B$, as well as a significant amplification of the segment of chromosome $6 p$ harboring the histone gene cluster containing these genes. Thus, mutations in histone $H 2 A / H 2 B$ genes were significantly enriched in carcinosarcomas (CSs) compared with carcinomas (mutations in $21.2 \%$ of CSs and $5.2 \%$ of uterine and ovarian epithelial tumor). These findings implicate mutations in histone $H 2 A / H 2 B$ genes in ECS.

Le Gallo et al. [40] have reported forkhead box A2 (FOXA2) mutations in 15.1\% of ECS. FOXA2 had not previously been implicated in ECSs and was predominated by frameshift and nonsense mutations. Sequencing of FOXA2 in 160 primary endometrial carcinomas revealed somatic mutations in $5.7 \%$ of serous, $22.7 \%$ of clear cell, $9 \%$ of endometrioid, and $11.1 \%$ of mixed endometrial carcinomas, the majority of which were frameshift mutations. Collectively, the findings of the study of Le Gallo et al. [40] provide evidence that FOXA2 is a pathogenic driver gene in the etiology of primary uterine cancers, including ECSs.

Similarly to ESC, ECS is characterized by aneuploidy and a high frequency of copy number variations (CNVs). Analysis of ploidy and whole-genome doubling has established a median ploidy of 3.3 and that $90 \%$ of ECS had undergone at least one whole-genome-doubling event. This percentage is 
significantly higher than in serous ovarian tumors, the tumor type with the next highest frequency of genomic doubling in the TCGA [38].

Recurring focal amplifications reported in the TCGA [38], some of which have also been observed in other series [51], include those containing known oncogenes such as TERC (3q26.2), FGFR3 (4p16.3), MYC (8q24.21), KAT6A (10q22.2), MDM2 (12q15), ERBB2 (17q12), CCND1 (11q13), CCNE1 (19q12), BCL2L1 (20q11.21), and RIT1 (1q22) (Figure 3).

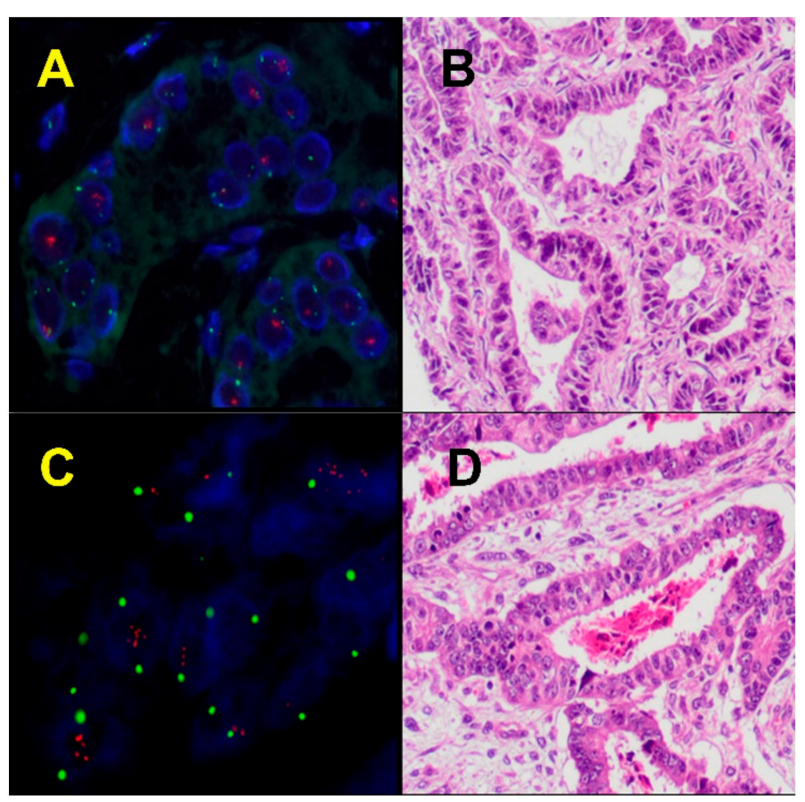

Figure 3. Amplification of oncogenes in endometrial carcinosarcomas analyzed by fluorescence in situ hybridization (FISH) (A) and (B), MYC proto-oncogene, bHLH transcription factor (MYC) amplification (C), and (D) Cyclin D1 (CCND1) amplification. Original magnification $\times 100$ for A and C, and $\times 20$ for B-D.

Cyclin D1 (CCNE1) is the most frequently amplified gene in ECS, 41\% according to data derived from TCGA (Table 1). In other tumors, for example, ovarian high-grade serous carcinoma, amplification of CCNE1 is associated with a worse prognosss and resistance to chemotherapy. According to Schipf et al., c-MYC amplification had a higher frequency in the carcinomatous compared to the sarcomatous tumor component. In their data on 30 carcinosarcomas of the ovary and uterus, $c-M Y C$ gene amplification was reported in $78 \%$ by fluorescence in situ hybridization (FISH) [52]. However, the TCGA data showed amplification of $c-M Y C$ in only $21 \%$ of ECSs [38].

The frequency of ERBB2 amplification in ECS ranged from 3-20\% [30,38,53-55]. Thus, ECS patients with ERBB2 amplification could benefit from anti-HER2 (human epidermal growth factor receptor 2) therapies, such as Trastuzumab. For patients unresponsive to chemotherapy and Trastuzumab, T-DM1 (Trastuzumab emtansine) may offer an alternative treatment option, as recent studies show how ECS cell lines and derived xenografts with ERBB2 amplification respond well to T-DM1 [56]. PIK3CA is amplified in 11\% of ECS, further highlighting the importance of the phosphatidylinositol 3-kinase (PIK3) pathway (see Section 4. Endometrioid-Like Molecular Alterations).

Schipf et al. detected ZNF217 amplification in $87 \%$ of gynecological CS [52]. Similarly to $c-M Y C$, in the TCGA data set the frequency is much lower $(9 \%)[28,38]$. Two other frequently amplified oncogenes in ECS, EGFR, and URI (unconventional prefolding RPB5 interactor 1), have not been found in the TCGA data set. Biscuola et al. reported EGFR amplification by FISH in 19\% of tumors [57], while in studies with smaller sample size, EGFR (epidermal growth factor receptor) protein overexpression has been reported in $45 \%$ to $82 \%$ of ECS, where a higher level of expression was seen in the sarcomatous component [53,58,59]. URI1 amplification has been reported in 40\% of ECS [60]. URI1 amplification 
was also associated with poor survival and reduced response to adjuvant treatment. Likewise, in a cultured cell model, overexpression of URI1 induced ATM (ATM Serine/Threonine Kinase) expression and resistance to cisplatin [60]. Recurring GPC5 (Glypican 5) gain/amplification has been detected in a subset of ECS, mostly in the sarcoma component, and the authors linked the involvement of GPC5 with sarcomatous transformation [61].

\section{Endometrioid-Like Molecular Alterations}

Mutations in genes encoding for the kinase or regulatory proteins of the PI3K/AKT (phosphatidylinositol 3-kinase/(Protein Kinase B) pathway have been detected in up to $67 \%$ of ECS [29]. Moreover, multiple PI3K/AKT pathway proteins have been found mutated in one tumor. PIK3CA mutations have been found in $11 \%$ to $40 \%[29,30,36,38,57,62,63]$ of the tumors. Unlike for TP53, with mutations concentrated on HotSpot regions, the mutations in PIK3CA are found scattered in the different functional domains. In addition to the traditional PIK3CA hotspots in exons 9 and 20, a smaller portion of ECS has mutations in exon 1, in the adaptor binding domain, helical domain, and C2 domain which increase kinase enzymatic activity [29,57].

The importance of mutations in this pathway comes from the fact that PI3KCA mutations have been detected in both the carcinoma and sarcoma components of the primary tumor and also in the metastatic tumor. This implies that they are important early events in the tumorigenesis of carcinosarcoma and thus could be targeted with PIK3CA/mTOR (Phosphatidylinositol-4,5-Bisphosphate 3-Kinase Catalytic Subunit Alpha /Mechanistic Target of Rapamycin Kinase) inhibitors [29,38]. PIK3CA inhibition has been applied successfully in advanced endometrial cancers [64].

Phosphatase And Tensin Homolog (PTEN) mutations are not as frequent as PIK3CA, but they are present in approximately $20 \%$ of ECS: $17 \%$ and $19 \%$ in the series reported by McConechy et al. [29] and the TCGA [38], respectively. However, Jones et al. reported that $47 \%$ of ECS carried PTEN mutation, but their series included only 17 cases [36]. PTEN and PIK3CA mutations frequently coexist in the same ECS [29].

Other genes with less frequency of mutations in the PI3K/AKT pathway in ECSs include Phosphatidylinositol 3-Kinase Regulatory Subunit 1 (PIK3R1) (10-17\%), PIK3R2, AKT1, AKT2, and AKT3 (less than $5 \%$ for each gene) [29,36,38,57].

AT-Rich Interaction Domain 1A (ARID1A) and Catenin Beta 1 (CTNNB1) are commonly mutated in EEC, and ARID1A mutations occur also in 10\% to $15 \%$ of ECS, leading usually to loss of of protein expression, while mutations in CTNNB1 are infrequent in ECS $[36,38,63]$. KRAS mutations were found in $12 \%$ and Cadherin 4 (CDH4) mutations in $18 \%$ [38].

Mismatch repair deficiency (MMR-def) and POLE mutations are more common in EEC than in ESC. MMR-def is due to germline or somatic even affecting mismatch repair genes, most frequently MutL Homolog 1 (MLH1), MutS Homolog 2 (MSH2), MutS Homolog 6 (MSH6), and Mismatch Repair Endonuclease PMS2 (PMS2). In sporadic EC, MMR-def is detected in 15-30\% of cases [65], although a higher frequency has been detected among high-grade endometrioid carcinomas (45-63\%) [31], most frequently due to $M L H 1$ promotor methylation. In addition, between $2-6 \%$ of endometrial carcinoma occurs in the context of Lynch syndrome due to germline mutations [66]. The frequency of MMR-def varies between 3\% and 23\% in ECS. The higher frequencies come from studies with a small sample size [36,67], while lower percentages have been observed in a bigger series [37,68]. MLH1 promoter methylation is probably the major cause for MMR-def in most tumors [68], and accordingly, MLH1 was epigenetically silenced in the two samples with MMR-def in the TCGA series [38].

Mutations in DNA Polymerase Epsilon, Catalytic Subunit (POLE) are present in some ECS, both of the most common HotSpot-mutations (P286R and V114L) have been identified in individual cases of ECS $[38,69,70]$. The most common mutations detected by NGS in recent studies are shown in Table 2 . 
Table 2. Comparison of gene mutation frequency among different series of Endometrial carcinosarcoma (ECS) analyzed by next-generation sequencing.

\begin{tabular}{|c|c|c|c|c|c|}
\hline Gene & Cherniack $(n=57)$ & McConechy $(n=30)$ & Jones $(n=361)$ & Zhao $(n=64) *$ & Le Gallo $(n=53)$ \\
\hline TP53 & $91 \%$ & $80 \%$ & $67 \%$ & $\sim 80 \%$ & $76 \%$ \\
\hline FBXW7 & $39 \%$ & $20 \%$ & & $\sim 22 \%$ & $19 \%$ \\
\hline PIKЗСА & $35 \%$ & $40 \%$ & $22 \%$ & $\sim 20 \%$ & $34 \%$ \\
\hline PPP2R1A & $28 \%$ & $13 \%$ & & $\sim 25 \%$ & $19 \%$ \\
\hline PTEN & $19 \%$ & $27 \%$ & & $\sim 7 \%$ & \\
\hline CHD4 & $17 \%$ & & & $\sim 20 \%$ & $17 \%$ \\
\hline ARID1A & $12 \%$ & $10 \%$ & & $\sim 4 \%$ & \\
\hline$K R A S$ & $12 \%$ & $10 \%$ & & $\sim 4 \%$ & \\
\hline PIK3R1 & $11 \%$ & $17 \%$ & & $\sim 4 \%$ & \\
\hline AKT3 & & $5 \%$ & & & \\
\hline BRCA1 & & & $18 \%$ & & \\
\hline$B R C A 2$ & & & $27 \%$ & & \\
\hline ZFHX3 & & $7 \%$ & & & \\
\hline CSMD3 & & $23 \%$ & & & \\
\hline HIST1H2BJ/G & & & & $21 \%$ & \\
\hline FOXA2 & & & & & $15 \%$ \\
\hline
\end{tabular}

* approximated \% in a combined series of endometrial and ovarian carcinosarcomas.

\section{Gene Expression Profiles in ECS}

Several studies have analyzed mRNA and microRNA (miRNA) expression profiles in ECS in comparison to other histological types of EC [71]. Regarding mRNA expression profiles, ECS differs from other EC histotypes in the expression, among others, of genes modulating epithelial-to-mesenchymal transition (EMT) and immune response (see Section 9. Immune Response in CS), and in the expression of cancer-testis antigens (CTA).

There are over 200 CTAs, which are classified into different families according to their sequence homology. In general, CTA genes are expressed only in normal testis and cancerous tissue. In many instances, CTA families are formed by clusters of nearly identical genes that are frequently located on the X-chromosome. A shared regulatory mechanism for related CTA clusters has been suggested as whole families of CTAs are often co-expressed together in tumors [72,73].

Overexpression of many members of the CTA family, such as melanoma antigen family A (MAGEA) members (MAGEA6, MAGEA9, MAGEA12), XAGE2, CTCFL, and CTAG1A (cancer/testis antigen 1A) has been reported in ECS [73]. CTCF, also known as the brother of the regulator of imprinted sites (BORIS), is an oncogene that deregulates the cancer epigenome, which is a common event in ECS [73,74]. Expression of CCCTC-Binding Factor Like (CTCFL) probably mediates the demethylation of another CTA gene, thus resulting in activation via repression [74]. Other genes of the CTA family associated with ECS, include, New York esophageal squamous cell carcinoma-1 (NY-ESO-1) and Preferentially Expressed Antigen In Melanoma (PRAME) [75,76]. Considering the tissue-restricted expression of CTA and its immunogenicity, immunotherapy based on CTA vaccines might be beneficial to ECS patients [73].

The miRNA signature of carcinosarcomas differs from endometrioid and serous carcinomas [77]. The function of miRNAs is to regulate gene expression by silencing. For this, they pair to the three prime untranslated region ( $3^{\prime}$ UTR) of the target mRNA sequence and thereby direct their posttranscriptional repression. miRNAs are small noncoding RNAs, which in turn can be regulated by promotor methylation and transcription factors, or by miRNA processing and stability [78]. 
In addition to miRNAs related to EMT (see Section 7. Epithelial-to-Mesenchymal Transition), miR-20b, miR-301, and miR-487 are up-regulated in carcinosarcomas compared to both endometrioid and serous tumors, whereas miR-518b is down-regulated. Low expression of miR-20b seems to inhibit tumor cell growth but then again help the tumor cell to gain resistance to apoptosis in hypoxic conditions [79]. In another study, miR-888 overexpression was detected in ECS, and the progesterone receptor was its direct target [80]. Finally, lower cancer-specific survival has been associated with upregulation of miR-184 and downregulation of let-7b-5p and miR-124 [81].

\section{Methylation Profiles in ECS}

Similarly to other types of cancers, ECS displays abnormal DNA methylation patterns including genome-wide hypomethylation and site-specific hypermethylation, associated with increased expression of DNA methyltransferases (DNMT1, DNMT3a), when compared to the normal endometrium [38,82]. Regarding global hypomethylation, Li et al. [82] reported that in normal endometrium, the $80 \%$ of analyzed CpGs were methylated, whereas, in ECS samples, this ratio fell to $60 \%$ to $70 \%$. In addition, all major classes of genomic transposable elements exhibited global DNA hypomethylation in ECS, with Long interspersed nuclear elements (LINEs) exhibiting the largest effect size. This effect was greater in ECS than in other histological types of endometrial carcinomas.

A number of tumor suppressor genes with recurrent hypermethylated promoters has also been reported in ECS, KLF4, NDN, WT1, PROX1, among others. Promoter hypermethylation of these genes is also common in other types of EC [38,82]. Interestingly, Cherniak et al. [38] reported that unsupervised cluster analysis of DNA methylation profiles of ECS grouped the tumors into three main classes according to their cancer-specific hypermethylation patterns. One group of tumors exhibited a hypermethylation pattern similar to that of EEC, whereas the others were much more similar to the ESC. Accordingly, the frequency of PTEN mutations was higher in the first group.

A constant characteristic of ECS is the aberrant DNA methylation of miR-200 genes (see discussion in Section 7. Epithelial-to-Mesenchymal Transition).

\section{Epithelial-to-Mesenchymal Transition}

EMT is a biological process that involves the acquisition of a mesenchymal/stem-cell-like phenotype by the (malignant) epithelial cells, endowing these cells with migratory and invasive properties, promoting cancer progression, preventing cell death and senescence, and inducing resistance to chemotherapy [83]. EMT has an important role in cancer, especially in tumor invasion and metastasis. During EMT, epithelial cells undergo a "cadherin switch" in which expression of N-cadherin is increased and E-cadherin expression reduced. E-cadherin can be repressed by either zinc-finger transcription factors (Snail1 (SNAI1), Slug/Snail2 (SNAI2), ZEB2 (SIP1) and ZEB1 ( $\delta$-EF1)) or basic helix-loop-helix transcription factors (E47 (TCF3), E2-2 (TCF4) or Twist). These EMT transcription factors (EMT-TF) can become activated through activation of different pathways such as Transforming Growth Factor Beta 1 (TGF $\beta$ ), tyrosine kinase receptors and Wnt, among others [25].

We have previously suggested that EMT is activated in ECS $[65,73,84,85]$. Further studies have confirmed this suggestion $[25,38,39,84,86]$. For example, we used real-time PCR to measure the differences in the expression of, E-cadherin, cadherin-11, SPARC, SNAIL, ZEB1, ZEB2, TWIST-1, TCF4, TGF $\beta 1$, and TGF $\beta 2$ between the epithelial and mesenchymal components of 23 ECSs. Also, we used immunohistochemistry to evaluate the expression of E-, P- and N-cadherin, cadherin-11, p120, vimentin, SPARC, fascin, and caveolin-1 in 76 ECS. In the mesenchymal component, a "cadherin switch" from E-cadherin to $\mathrm{N}$-cadherin and cadherin 11 was observed. In addition, upregulation of all of E-cadherin repressors together with overexpression of all mesenchymal markers tested was demonstrated.

Also, High Mobility Group AT-Hook 2 (HMGA2) has a role in EMT as a regulator of SNAI1 expression and of other transcription factors downstream of SNAI1, such as Slug, ZEB1, and ZEB2. HMGA2 has been proposed to be regulated by the let-7/Lin28B pathway. Accordingly, we have 
previously demonstrated that an increase of Lin28B expression correlated with let-7b down-regulation and HMGA2 overexpression in ECS [73].

A role of the WNT pathway in the transition from an epithelial to a mesenchymal status is demonstrated by the fact that up to $23 \%$ of ECS showed nuclear $\beta$-catenin, not associated with CTNNB1 mutation, in the sarcomatous but not in the carcinomatous component [57]. Nuclear $\beta$-catenin cooperates with Sox4 and p300 to transcriptionally up-regulate Slug to induce EMT [87].

Similarly to $\beta$-catenin, in another study, ALK tyrosine kinase receptor (ALK) was frequently over-expressed in the sarcomatous components of EC [87]. The authors suggest that ALK-related cascades could participate in divergent sarcomatous differentiation through the induction of EMT and inhibition of apoptosis [87]. In contrast, although the expression of L1CAM is a strong predictor of poor outcome in endometrial cancer and overexpression of L1CAM has been related to EMT in endometrial cancer cell lines [88], in clinical samples of ECS, only the epithelial component was positive in $65 \%$ of the cases, while no expression was seen in the mesenchymal part. Thus in ECS, L1CAM is not a marker for the mesenchymal phenotype [89].

MicroRNA signatures associated with EMT and their relationships with EMT markers in human carcinosarcomas have been studied by us and more recently by Cherniack et al. [25,38,84]. We used real-time PCR to measure the differences in the expression of 384 miRNAs, between the epithelial and mesenchymal components of ECS and found that miR-200 family members were down-regulated in the mesenchymal part of the ECS. The miR-200 family plays a major role in regulating epithelial plasticity, mainly through its involvement in double-negative feedback loops with the EMT-TFs ZEB1, ZEB2, SNAI1, and SNAI2, ultimately influencing E-cadherin expression levels $[25,84,85]$. Down-regulation of miR-200 family members in ECs is not only due to the transcriptional repression by EMT-TF, but also to promoter methylation [38,84]. In this sense, experimental studies have demonstrated a major role of ZEB1 in transcriptional repression and of SNAI1 and, to a lesser extent, SNAI2 in the epigenetic silencing through DNA hypermethylation of miR-200 genes [84]. Other down-regulated miRNAs in our studies included miR-23b and miR-29c, involved in the inhibition of mesenchymal markers, and miR-203 and miR-205 involved in the inhibition of cell stemness [25,84].

\section{Beyond EMT: Stemness and Differentiation in ECS}

It has been demonstrated that epithelial cells undergoing EMT to acquire mesenchymal features are more likely to possess stemness. In addition, some studies suggested that stemness can be associated with cells undergoing a partial EMT and showing a hybrid Epithelial/Mesenchymal phenotype. Jolly et al. postulated that the core EMT and stemness modules, miR-200/ZEB and Lin28/let7, govern EMT decision making [90]. According to this hypothesis, not only the miR-200/ZEB EMT module is active in ECS, as previously discussed, but also, we have previously demonstrated that the expression of the suppressor of miRNA biogenesis Lin28B was increased in ECS when compared with EEC samples (62.85-fold change). Moreover, we observed a significant inverse correlation between the expression of Lin28B and let-7b, supporting the hypothesis that they participate in the same regulatory pathway [73].

Cells with an Epithelial/Mesenchymal hybrid phenotype evolve to an epithelial or a mesenchymal phenotype depending on factors acting on the EMT and stemness modules [91]. Both routes would enable a secondary round of differentiation to specific epithelial or mesenchymal phenotypes [92]. ECS exemplified well this hypothesis since different types of mesenchymal tissues could develop. This is illustrated not only by the morphological evidence of striated muscle, cartilage, or bone tissue in ECS but also by molecular evidence. Thus, the presence of rhabdomyoblastic differentiation in ECS, the most common heterologous mesenchymal differentiation in ECS, is accompanied by the overexpression of genes that are characteristic of primary embryonic myocytes [93]. Romero-Perez et al. [73] demonstrated that in ECS there was an overexpression of the core network of transcription factors that control the myogenic program in primary myocytes, including Myf5, Myf6, MyoD, and MYOG (myogenin), in addition to other transcriptional factors involved in this process, such as SIX1 and EYE1/2. Moreover, 
overexpression of genes encoding specialized cytoskeletal proteins, such as slow (Myh7) and embryonic (Myh3) myosin heavy chains and skeletal $\alpha$-actin (Acta1), was also observed. Similar to our previous results, Lu et al. [94] reported that 18 out of 57 ECS reported in the TCGA had a gene expression pattern enriched in genes involved in muscle development and morphogenesis, myoblast differentiation, and contraction regulation.

\section{Immune Response in CS}

The tumor microenvironment has an important role in cancer and immunomodulation of the microenvironment is a new focus in cancer medicine [95]. Accumulated evidence indicates that ECS is a rational target for immune therapy. In their study of gene expression, Romero-Peréz et al. found that over 10\% of the genes differentially expressed between ECS and EEC were implicated in the immune response, suggesting differential immunomodulation between histotypes [73].

Ayers et al. have created a Tumor Inflammation Signature (TIS) using gene expression data from baseline tumor samples of pembrolizumab-treated patients. The signature includes 18 genes that reflect a suppressed adaptive immune response (antigen presentation, chemokine expression, cytotoxic activity, and adaptive immune resistance) and is enriched in tumors with sensitivity to Programmed cell death 1 ligand 1 (PD-L1) inhibitors [96]. In another study, Danaher et al. concluded that, although there was only a correlation between TIS and tumor mutational burden (TMB), the tumors could be classified equally well with either TIS or TMB [97]. Using data from TCGA, we compared the TIS between endometrioid and serous endometrial and ECS and observed that it is significantly lower in uterine carcinosarcoma ECS compared to both ECS and ESC (analysis of variance (ANOVA), p < 0.001, Figure 4). However, TIS varies more within than between tumor types, and although ECS has a relatively low score on average, more samples need to be studied to see if a group of patients might show association with prognosis or immunotherapy response prediction. For example in breast cancer, patients with the highest $10 \%$ of the TIS score had a markedly better prognosis [97].

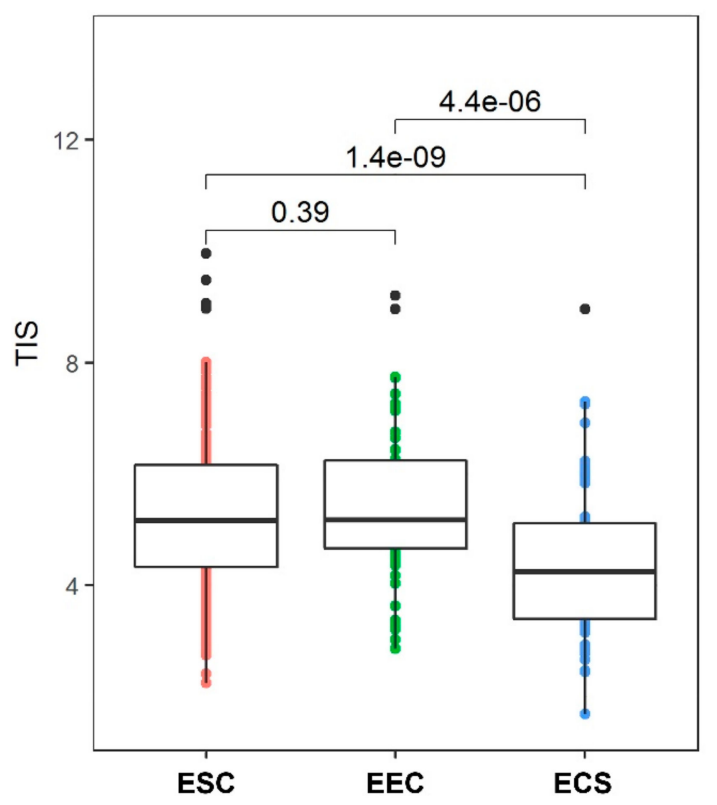

Figure 4. A boxplot histogram of Tumor Inflammation Signature (TIS) scores by endometrial cancer type in endometrial carcinosarcoma (ECS), endometrial serous carcinoma (ESC), and endometrial endometrioid carcinoma (EEC). $p$ values from analysis of variance (ANOVA) test are shown for all comparisons. 
Several studies have demonstrated that EMT contributes to evasion of immune surveillance [98-105]. PD-L1 has a major role in tumor immune escape and also in the development of a permissive immune microenvironment [105]. Different studies have observed an association between PD-L1 expression and mesenchymal characteristics in different tumor types, such as breast, lung, and pancreatic adenocarcinomas, among others. Also, it has been shown that miR-200 targets PD-L1. Moreover, the EMT-TF ZEB1 relieves miR-200 repression of PD-L1 on tumor cells, leading to CD8(+) T-cell immunosuppression and metastasis.

Regarding carcinosarcomas, PD-L1 expression was significantly higher in lung carcinosarcoma than in conventional non-small-cell lung carcinoma [106], providing a rationale for the potential use of immunotherapy. In this sense, a significant benefit of Nivolumab treatment in PD-L1 positive metastatic pulmonary carcinosarcoma has been reported in some patients [107]. In ovarian carcinosarcoma, PD-L1-positive expression was also observed in about $50 \%$ of the tumors, without differences between the epithelial and mesenchymal components [108]. To the best of our knowledge, there are only two studies on PD-L1 expression in ECS. Whereas in one study, PD-L1 was expressed in $25 \%$ of the tumors [30], in another, up to $86 \%$ of ECS expressed the biomarker [109]. This subset of tumors could benefit from drugs directed to the PD-1/PD-L1 pathway.

\section{Conclusions and Perspectives}

Carcinosarcoma is a heterogeneous aggressive endometrial carcinoma that probably represents the end-stage of the evolution of both endometrioid and serous carcinomas after triggering a stable EMT program (Figure 5). Molecular observations suggest that, although infrequent, endometrioid carcinomas associated with mutations in PTEN or PIK3CA are more prone to acquire TP53 mutations than those associated with MMR-def, POLE, or CTNNB1 mutations. Mutations in TP53 seem to be essential, but not sufficient, to ECS development, since they are as frequent in ECS as in endometrial serous carcinoma. Although it is not clear what triggers EMT in tumors with TP53 mutation, a common characteristic of all ECS is the switching of cadherins, the overexpression EMT-TF, and the down-regulation of miR-200 genes. Probably, the crosstalk of different EMT-TF and the differential regulation of miR-200 genes by transcriptional repression or by epigenetic silencing through DNA hypermethylation play a major role in fixing the mesenchymal phenotype. Subsequent activation of specific transcription programs could induce differentiation to diverse mesenchymal tissues.

At present, most patients with ECS are not stratified for treatment according to molecular alterations [110,111]. However, future clinical trials will most likely take into account this data. For example, a recent report has demonstrated the benefit provided when Traztuzumab is included in the treatment of ESC with HER2 amplification [112]. Considering the similarities between ESC and ECS, it is reasonable to think that anti-HER2 therapies would also benefit patients with HER2-positive ECS. Although the relatively low-frequency of ECS hinders efforts to design specific clinical trials, there are promising areas of research, such as the use of immunotherapy in tumors with POLE mutations, MMR-def, and high TMB, and also the use of Poly (ADP-ribose) polymerase (PARP) inhibitors in tumors with homologous recombination deficiency, especially due to germline or somatic BRCA mutations. 

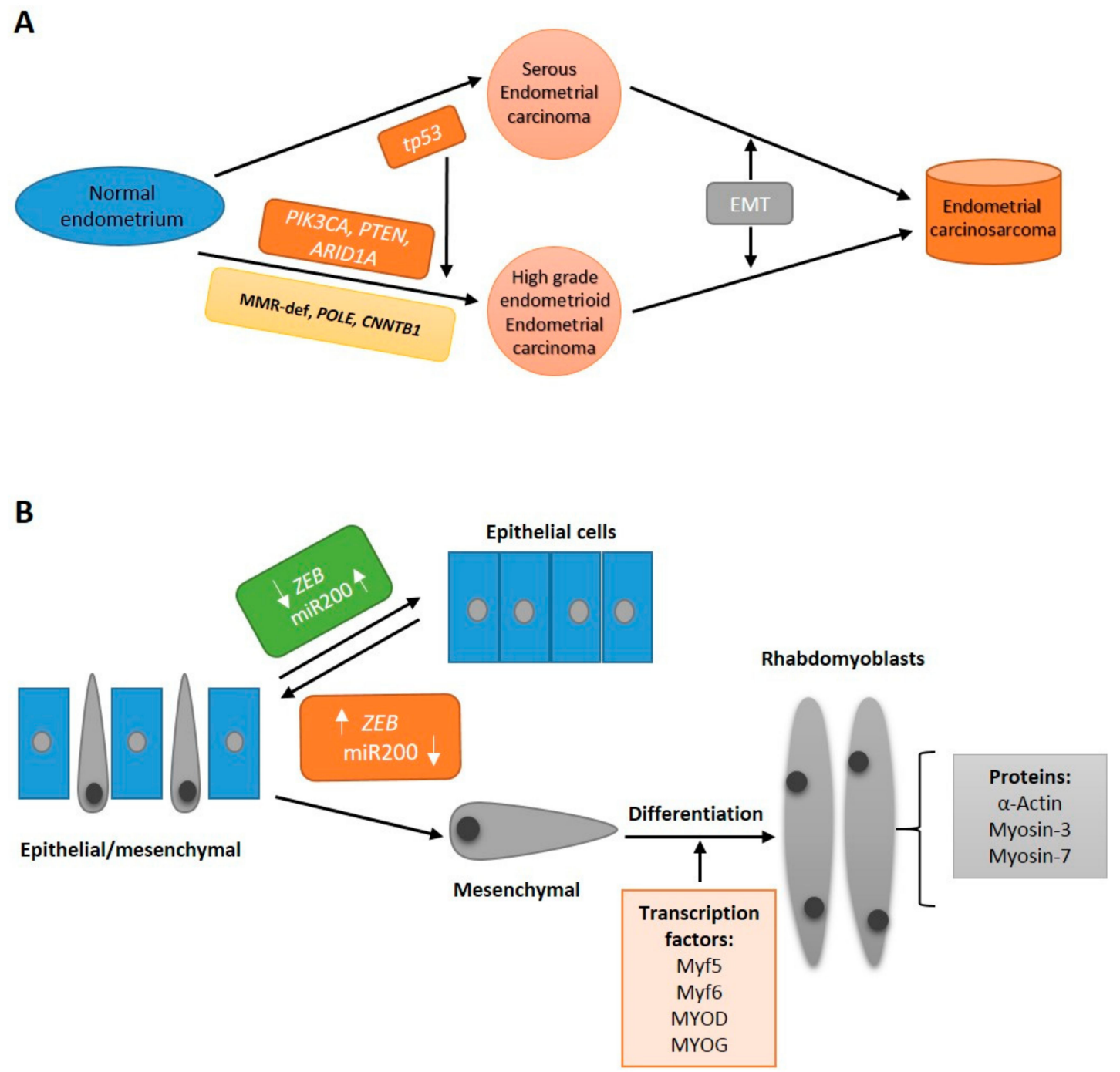

Figure 5. A proposed model of development of endometrial carcinosarcoma. (A) Evolution of both endometrioid and serous carcinomas to endometrial carcinosarcoma after eliciting a stable epithelial-to-mesenchymal transition (EMT) program. Transformation of normal endometrium to serous endometrial carcinoma is triggered by mutation in TP53. Endometrioid carcinomas with mutations in genes of the phosphatidylinositol 3-kinase (PIK3) pathway or ARID1A are more prone to acquire TP53 mutations than those with mismatch repair deficiency or mutations in POLE and CTNNB1. (B) Endometrial carcinosarcomas are composed by a mixed population of cells representing diverse EMT states. The relative expression of some factors, such as miR-200 or ZEBs, dictate the specific cell state: epithelial, hybrid, or mesenchymal (adapted from Ref. 92).

Author Contributions: Conceptualization, S.L., and J.P.; Data curation, E.C. and J.M.R.-R.; Formal analysis, J.M.R.-R., S.F.O. and X.M.G.-G.; Funding acquisition, J.P.; Resources, J.P.; Supervision, B.P.-M., X.M.G.-G. and J.P.; Writing-original draft, S.L., B.P.-M., M.B., M.L.P.-B. and J.P.; Writing-review \& editing, S.L., X.M.G.-G. and J.P.

Funding: This review was funded by grants from the Instituto de Salud Carlos III (ISCIII) (PIE15/00050 and PI16/00887) and CIBERONC (CB16/12/00316 and CB16/12/00231, CB16/12/00361), co-financed by the European Development Regional Fund. 'A way to achieve Europe' (FEDER), and by the Spanish Association Against Cancer Scientific Foundation (grants: AIO-aecc 2016 and Grupos Coordinados Traslacionales aecc 2018).

Conflicts of Interest: The authors declare no conflict of interest. The funders had no role in the design of the study; in the collection, analyses, or interpretation of data; in the writing of the manuscript, or in the decision to publish the results. 


\section{References}

1. Tavassoli, F. World Health Organization Classification of Tumours. Pathology and Genetics of Tumors of Breast and Female Genital Organs; IARC Press: Lyon, France, 2003.

2. Gonzalez Bosquet, J.; Terstriep, S.A.; Cliby, W.A.; Brown-Jones, M.; Kaur, J.S.; Podratz, K.C.; Keeney, G.L. The impact of multi-modal therapy on survival for uterine carcinosarcomas. Gynecol. Oncol. 2010, 116, 419-423. [CrossRef] [PubMed]

3. De Jong, R.A.; Nijman, H.W.; Wijbrandi, T.F.; Reyners, A.K.; Boezen, H.M.; Hollema, H. Molecular markers and clinical behavior of uterine carcinosarcomas: Focus on the epithelial tumor component. Mod. Pathol. 2011, 24, 1368-1379. [CrossRef] [PubMed]

4. Ferguson, S.E.; Tornos, C.; Hummer, A.; Barakat, R.R.; Soslow, R.A. Prognostic features of surgical stage I uterine carcinosarcoma. Am. J. Surg. Pathol. 2007, 31, 1653-1661. [CrossRef] [PubMed]

5. Amant, F.; Cadron, I.; Fuso, L.; Berteloot, P.; de Jonge, E.; Jacomen, G.; Van Robaeys, J.; Neven, P.; Moerman, P.; Vergote, I. Endometrial carcinosarcomas have a different prognosis and pattern of spread compared to high-risk epithelial endometrial cancer. Gynecol. Oncol. 2005, 98, 274-280. [CrossRef] [PubMed]

6. George, E.; Lillemoe, T.J.; Twiggs, L.B.; Perrone, T. Malignant mixed mullerian tumor versus high-grade endometrial carcinoma and aggressive variants of endometrial carcinoma: A comparative analysis of survival. Int. J. Gynecol. Pathol. 1995, 14, 39-44. [CrossRef] [PubMed]

7. Callister, M.; Ramondetta, L.M.; Jhingran, A.; Burke, T.W.; Eifel, P.J. Malignant mixed mullerian tumors of the uterus: Analysis of patterns of failure, prognostic factors, and treatment outcome. Int. J. Radiat. Oncol. Biol. Phys. 2004, 58, 786-796. [CrossRef]

8. Bansal, N.; Herzog, T.J.; Seshan, V.E.; Schiff, P.B.; Burke, W.M.; Cohen, C.J.; Wright, J.D. Uterine carcinosarcomas and grade 3 endometrioid cancers: Evidence for distinct tumor behavior. Obstet. Gynecol. 2008, 112, 64-70. [CrossRef] [PubMed]

9. Matsuo, K.; Ross, M.S.; Machida, H.; Blake, E.A.; Roman, L.D. Trends of uterine carcinosarcoma in the United States. J. Gynecol. Oncol. 2018, 29, e22. [CrossRef] [PubMed]

10. Jeong, B.K.; Sung, C.O.; Kim, K.R. Uterine malignant mixed mullerian tumors following treatment with selective estrogen receptor modulators in patients with breast cancer: A report of 13 cases and their clinicopathologic characteristics. J. Pathol. Transl. Med. 2019, 53, 31-39. [CrossRef]

11. Matsuo, K.; Ross, M.S.; Bush, S.H.; Yunokawa, M.; Blake, E.A.; Takano, T.; Ueda, Y.; Baba, T.; Satoh, S.; Shida, M.; et al. Tumor characteristics and survival outcomes of women with tamoxifen-related uterine carcinosarcoma. Gynecol. Oncol. 2017, 144, 329-335. [CrossRef]

12. Buza, N.; Tavassoli, F.A. Comparative analysis of P16 and P53 expression in uterine malignant mixed mullerian tumors. Int. J. Gynecol. Pathol. 2009, 28, 514-521. [CrossRef] [PubMed]

13. Silverberg, S.G.; Major, F.J.; Blessing, J.A.; Fetter, B.; Askin, F.B.; Liao, S.Y.; Miller, A. Carcinosarcoma (malignant mixed mesodermal tumor) of the uterus. A gynecologic oncology group pathologic study of 203 cases. Int. J. Gynecol. Pathol. 1990, 9, 1-19. [CrossRef] [PubMed]

14. Sreenan, J.J.; Hart, W.R. Carcinosarcomas of the female genital tract. A pathologic study of 29 metastatic tumors: Further evidence for the dominant role of the epithelial component and the conversion theory of histogenesis. Am. J. Surg. Pathol. 1995, 19, 666-674. [CrossRef] [PubMed]

15. Costa, M.J.; Vogelsan, J.; Young, L.J. p53 gene mutation in female genital tract carcinosarcomas (malignant mixed mullerian tumors): A clinicopathologic study of 74 cases. Mod. Pathol. 1994, 7, 619-627. [PubMed]

16. Matsuo, K.; Takazawa, Y.; Ross, M.S.; Elishaev, E.; Podzielinski, I.; Yunokawa, M.; Sheridan, T.B.; Bush, S.H.; Klobocista, M.M.; Blake, E.A.; et al. Significance of histologic pattern of carcinoma and sarcoma components on survival outcomes of uterine carcinosarcoma. Ann. Oncol. 2016, 27, 1257-1266. [CrossRef] [PubMed]

17. Abdulfatah, E.; Lordello, L.; Khurram, M.; Van de Vijver, K.; Alosh, B.; Bandyopadhyay, S.; Oliva, E.; Ali-Fehmi, R. Predictive histologic factors in carcinosarcomas of the uterus: A multiinstitutional study. Int. J. Gynecol. Pathol. 2018, 38, 205-215. [CrossRef]

18. Costa, M.J.; Khan, R.; Judd, R. Carcinoma (malignant mixed mullerian [mesodermal] tumor) of the uterus and ovary. Correlation of clinical, pathologic, and immunohistochemical features in 29 cases. Arch. Pathol. Lab. Med. 1991, 115, 583-590. [PubMed] 
19. Kurnit, K.C.; Previs, R.A.; Soliman, P.T.; Westin, S.N.; Klopp, A.H.; Fellman, B.M.; Lu, K.H.; Ramondetta, L.M.; Fleming, N.D. Prognostic factors impacting survival in early stage uterine carcinosarcoma. Gynecol. Oncol. 2019, 152, 31-37. [CrossRef]

20. McCluggage, W.G. A practical approach to the diagnosis of mixed epithelial and mesenchymal tumours of the uterus. Mod. Pathol. 2016, 29 (Suppl. 1), S78-S91. [CrossRef]

21. Matsuo, K.; Takazawa, Y.; Ross, M.S.; Elishaev, E.; Yunokawa, M.; Sheridan, T.B.; Bush, S.H.; Klobocista, M.M.; Blake, E.A.; Takano, T.; et al. Significance of lymphovascular space invasion by the sarcomatous component in uterine carcinosarcoma. Ann. Surg. Oncol. 2018, 25, 2756-2766. [CrossRef]

22. Iwasa, Y.; Haga, H.; Konishi, I.; Kobashi, Y.; Higuchi, K.; Katsuyama, E.; Minamiguchi, S.; Yamabe, H. Prognostic factors in uterine carcinosarcoma: A clinicopathologic study of 25 patients. Cancer 1998, 82, 512-519. [CrossRef]

23. Matsuo, K.; Takazawa, Y.; Ross, M.S.; Elishaev, E.; Yunokawa, M.; Sheridan, T.B.; Bush, S.H.; Klobocista, M.M.; Blake, E.A.; Takano, T.; et al. Characterizing sarcoma dominance pattern in uterine carcinosarcoma: Homologous versus heterologous element. Surg. Oncol. 2018, 27, 433-440. [CrossRef] [PubMed]

24. Matsuo, K.; Takazawa, Y.; Ross, M.S.; Elishaev, E.; Yunokawa, M.; Sheridan, T.B.; Bush, S.H.; Klobocista, M.M.; Blake, E.A.; Takano, T.; et al. Proposal for a risk-based categorization of uterine carcinosarcoma. Ann. Surg. Oncol. 2018, 25, 3676-3684. [CrossRef] [PubMed]

25. Castilla, M.A.; Moreno-Bueno, G.; Romero-Perez, L.; Van De Vijver, K.; Biscuola, M.; López-García, M.Á.; Prat, J.; Matías-Guiu, X.; Cano, A.; Oliva, E.; et al. Micro-RNA signature of the epithelial-mesenchymal transition in endometrial carcinosarcoma. J. Pathol. 2011, 223, 72-80. [CrossRef] [PubMed]

26. Chiyoda, T.; Tsuda, H.; Tanaka, H.; Kataoka, F.; Nomura, H.; Nishimura, S.; Takano, M.; Susumu, N.; Saya, H.; Aoki, D. Expression profiles of carcinosarcoma of the uterine corpus-are these similar to carcinoma or sarcoma? Genes Chromosomes Cancer 2012, 51, 229-239. [CrossRef] [PubMed]

27. Wada, H.; Enomoto, T.; Fujita, M.; Yoshino, K.; Nakashima, R.; Kurachi, H.; Haba, T.; Wakasa, K.; Shroyer, K.R.; Tsujimoto, M.; et al. Molecular evidence that most but not all carcinosarcomas of the uterus are combination tumors. Cancer Res. 1997, 57, 5379-5385. [PubMed]

28. Talhouk, A.; McConechy, M.K.; Leung, S.; Li-Chang, H.H.; Kwon, J.S.; Melnyk, N.; Yang, W.; Senz, J.; Boyd, N.; Karnezis, A.N.; et al. A clinically applicable molecular-based classification for endometrial cancers. $\mathrm{Br}$. J. Cancer 2015, 113, 299-310. [CrossRef] [PubMed]

29. McConechy, M.K.; Hoang, L.N.; Chui, M.H.; Senz, J.; Yang, W.; Rozenberg, N.; Mackenzie, R.; McAlpine, J.N.; Huntsman, D.G.; Clarke, B.A.; et al. In-depth molecular profiling of the biphasic components of uterine carcinosarcomas. J. Pathol. Clin. Res. 2015, 1, 173-185. [CrossRef] [PubMed]

30. Jones, N.L.; Xiu, J.; Chatterjee-Paer, S.; Buckley de Meritens, A.; Burke, W.M.; Tergas, A.I.; Wright, J.D.; Hou, J.Y. Distinct molecular landscapes between endometrioid and nonendometrioid uterine carcinomas. Int. J. Cancer 2017, 140, 1396-1404. [CrossRef] [PubMed]

31. Rosa-Rosa, J.M.; Leskela, S.; Cristobal-Lana, E.; Santón, A.; López-García, M.Á.; Muñoz, G.; Pérez-Mies, B.; Biscuola, M.; Prat, J.; Esther, O.E.; et al. Molecular genetic heterogeneity in undifferentiated endometrial carcinomas. Mod. Pathol. 2016, 29, 1390. [CrossRef] [PubMed]

32. Kounelis, S.; Jones, M.W.; Papadaki, H.; Bakker, A.; Swalsky, P.; Finkelstein, S.D. Carcinosarcomas (malignant mixed mullerian tumors) of the female genital tract: Comparative molecular analysis of epithelial and mesenchymal components. Hum. Pathol. 1998, 29, 82-87. [CrossRef]

33. Abeln, E.C.; Smit, V.T.; Wessels, J.W.; de Leeuw, W.J.; Cornelisse, C.J.; Fleuren, G.J. Molecular genetic evidence for the conversion hypothesis of the origin of malignant mixed mullerian tumours. J. Pathol. 1997, 183, 424-431. [CrossRef]

34. Liu, F.S.; Kohler, M.F.; Marks, J.R.; Bast, R.C., Jr.; Boyd, J.; Berchuck, A. Mutation and overexpression of the p53 tumor suppressor gene frequently occurs in uterine and ovarian sarcomas. Obstet. Gynecol. 1994, 83, 118-124. [PubMed]

35. Kanthan, R.; Senger, J.L.; Diudea, D. Malignant mixed Mullerian tumors of the uterus: Histopathological evaluation of cell cycle and apoptotic regulatory proteins. World J. Surg. Oncol. 2010, 8, 60. [CrossRef] [PubMed]

36. Jones, S.; Stransky, N.; McCord, C.L.; Cerami, E.; Lagowski, J.; Kelly, D.; Angiuoli, S.V.; Sausen, M.; Kann, L.; Shukla, M.; et al. Genomic analyses of gynaecologic carcinosarcomas reveal frequent mutations in chromatin remodelling genes. Nat. Commun. 2014, 5, 5006. [CrossRef] [PubMed] 
37. McConechy, M.K.; Ding, J.; Cheang, M.C.; Wiegand, K.; Senz, J.; Tone, A.; Yang, W.; Prentice, L.; Tse, K.; Zeng, T.; et al. Use of mutation profiles to refine the classification of endometrial carcinomas. J. Pathol. 2012, 228, 20-30. [CrossRef] [PubMed]

38. Cherniack, A.D.; Shen, H.; Walter, V.; Stewart, C.; Murray, B.A.; Bowlby, R.; Hu, X.; Ling, S.; Soslow, R.A.; Broaddus, R.R.; et al. Integrated molecular characterization of uterine carcinosarcoma. Cancer Cell 2017, 31, 411-423. [CrossRef]

39. Liu, Y.; Weber, Z.; San Lucas, F.A.; Deshpande, A.; Jakubek, Y.A.; Sulaiman, R.; Fagerness, M.; Flier, N.; Sulaiman, J.; Davis, C.M.; et al. Assessing inter-component heterogeneity of biphasic uterine carcinosarcomas. Gynecol. Oncol. 2018, 151, 243-249. [CrossRef]

40. Le Gallo, M.; Rudd, M.L.; Urick, M.E.; Hansen, N.F.; National Institutes of Health Intramural Sequencing Center Comparative Sequencing Program; Merino, M.J.; Mutch, D.G.; Goodfellow, P.J.; Mullikin, J.C.; Bell, D.W. The FOXA2 transcription factor is frequently somatically mutated in uterine carcinosarcomas and carcinomas. Cancer 2018, 124, 65-73. [CrossRef]

41. D'Brot, A.; Kurtz, P.; Regan, E.; Jakubowski, B.; Abrams, J.M. A platform for interrogating cancer-associated p53 alleles. Oncogene 2017, 36, 286-291. [CrossRef]

42. Semczuk, A.; Skomra, D.; Chyzynska, M.; Szewczuk, W.; Olcha, P.; Korobowicz, E. Immunohistochemical analysis of carcinomatous and sarcomatous components in the uterine carcinosarcoma: A case report. Pathol. Res. Pract. 2008, 204, 203-207. [CrossRef] [PubMed]

43. Reid-Nicholson, M.; Iyengar, P.; Hummer, A.J.; Linkov, I.; Asher, M.; Soslow, R.A. Immunophenotypic diversity of endometrial adenocarcinomas: Implications for differential diagnosis. Mod. Pathol. 2006, 19, 1091-1100. [CrossRef] [PubMed]

44. Robinson-Bennett, B.; Belch, R.Z.; Han, A.C. Loss of p16 in recurrent malignant mixed mullerian tumors of the uterus. Int. J. Gynecol. Cancer 2006, 16, 1354-1357. [PubMed]

45. Saule, C.; Mouret-Fourme, E.; Briaux, A.; Becette, V.; Rouzier, R.; Houdayer, C.; Stoppa-Lyonnet, D. Risk of serous endometrial carcinoma in women with pathogenic BRCA1/2 variant after risk-reducing salpingo-oophorectomy. J. Natl. Cancer Inst. 2018, 110, 213-215. [CrossRef] [PubMed]

46. De Jonge, M.M.; Mooyaart, A.L.; Vreeswijk, M.P.; de Kroon, C.D.; van Wezel, T.; van Asperen, C.J.; Smit, V.T.; Dekkers, O.M.; Bosse, T. Linking uterine serous carcinoma to BRCA1/2-associated cancer syndrome: A meta-analysis and case report. Eur. J. Cancer 2017, 72, 215-225. [CrossRef]

47. Shu, C.A.; Pike, M.C.; Jotwani, A.R.; Friebel, T.M.; Soslow, R.A.; Levine, D.A.; Nathanson, K.L.; Konner, J.A.; Arnold, A.G.; Bogomolniy, F.; et al. Uterine cancer after risk-reducing salpingo-oophorectomy without hysterectomy in women with BRCA mutations. JAMA Oncol. 2016, 2, 1434-1440. [CrossRef] [PubMed]

48. Sonoda, Y.; Saigo, P.E.; Federici, M.G.; Boyd, J. Carcinosarcoma of the ovary in a patient with a germline BRCA2 mutation: Evidence for monoclonal origin. Gynecol. Oncol. 2000, 76, 226-229. [CrossRef]

49. Carnevali, I.W.; Cimetti, L.; Sahnane, N.; Libera, L.; Cavallero, A.; Formenti, G.; Riva, C.; Tibiletti, M.G. Two cases of carcinosarcomas of the ovary involved in hereditary cancer syndromes. Int. J. Gynecol. Pathol. 2017, 36, 64-70. [CrossRef]

50. Ghilli, M.; Mariniello, D.M.; Fanelli, G.; Cascione, F.; Fontana, A.; Cristaudo, A.; Cilotti, A.; Caligo, A.M.; Manca, G.; Colizzi, L.; et al. Carcinosarcoma of the breast: An aggressive subtype of metaplastic cancer. Report of a rare case in a young BRCA-1 mutated woman. Clin. Breast Cancer 2017, 17, e31-e35. [CrossRef]

51. Zhao, S.; Bellone, S.; Lopez, S.; Thakral, D.; Schwab, C.; English, D.P.; Black, J.; Cocco, E.; Choi, J.; Zammataro, L.; et al. Mutational landscape of uterine and ovarian carcinosarcomas implicates histone genes in epithelial-mesenchymal transition. Proc. Natl. Acad. Sci USA 2016, 113, 12238-12243. [CrossRef]

52. Schipf, A.; Mayr, D.; Kirchner, T.; Diebold, J. Molecular genetic aberrations of ovarian and uterine carcinosarcomas-a CGH and FISH study. Virchows Arch. 2008, 452, 259-268. [CrossRef] [PubMed]

53. Livasy, C.A.; Reading, F.C.; Moore, D.T.; Boggess, J.F.; Lininger, R.A. EGFR expression and HER2/neu overexpression/amplification in endometrial carcinosarcoma. Gynecol. Oncol. 2006, 100, 101-106. [CrossRef] [PubMed]

54. Raspollini, M.R.; Susini, T.; Amunni, G.; Paglierani, M.; Taddei, A.; Marchionni, M.; Scarselli, G.; Taddei, G.L. COX-2, c-KIT and HER-2/neu expression in uterine carcinosarcomas: Prognostic factors or potential markers for targeted therapies? Gynecol. Oncol. 2005, 96, 159-167. [CrossRef] [PubMed] 
55. Sawada, M.; Tsuda, H.; Kimura, M.; Okamoto, S.; Kita, T.; Kasamatsu, T.; Yamada, T.; Kikuchi, Y.; Honjo, H.; Matsubara, O. Different expression patterns of KIT, EGFR, and HER-2 (c-erbB-2) oncoproteins between epithelial and mesenchymal components in uterine carcinosarcoma. Cancer Sci. 2003, 94, 986-991. [CrossRef]

56. Nicoletti, R.; Lopez, S.; Bellone, S.; Cocco, E.; Schwab, C.L.; Black, J.D.; Centritto, F.; Zhu, L.; Bonazzoli, E.; Buza, N.; et al. T-DM1, a novel antibody-drug conjugate, is highly effective against uterine and ovarian carcinosarcomas overexpressing HER2. Clin. Exp. Metastasis 2015, 32, 29-38. [CrossRef] [PubMed]

57. Biscuola, M.; Van de Vijver, K.; Castilla, M.A.; Romero-Pérez, L.; López-García, M.Á.; Díaz-Martín, J.; Matias-Guiu, X.; Oliva, E.; Palacios Calvo, J. Oncogene alterations in endometrial carcinosarcomas. Hum. Pathol. 2013, 44, 852-859. [CrossRef] [PubMed]

58. Cimbaluk, D.; Rotmensch, J.; Scudiere, J.; Gown, A.; Bitterman, P. Uterine carcinosarcoma: Immunohistochemical studies on tissue microarrays with focus on potential therapeutic targets. Gynecol. Oncol. 2007, 105, 138-144. [CrossRef] [PubMed]

59. Hayes, M.P.; Douglas, W.; Ellenson, L.H. Molecular alterations of EGFR and PIK3CA in uterine serous carcinoma. Gynecol. Oncol. 2009, 113, 370-373. [CrossRef] [PubMed]

60. Wang, Y.; Garabedian, M.J.; Logan, S.K. URI1 amplification in uterine carcinosarcoma associates with chemo-resistance and poor prognosis. Am. J. Cancer Res. 2015, 5, 2320-2329. [PubMed]

61. Chui, M.H.; Have, C.; Hoang, L.N.; Shaw, P.; Lee, C.H.; Clarke, B.A. Genomic profiling identifies GPC5 amplification in association with sarcomatous transformation in a subset of uterine carcinosarcomas. J. Pathol. Clin. Res. 2018, 4, 69-78. [CrossRef]

62. Murray, S.; Linardou, H.; Mountzios, G.; Manoloukos, M.; Markaki, S.; Eleutherakis-Papaiakovou, E.; Dimopoulos, M.A.; Papadimitriou, C.A. Low frequency of somatic mutations in uterine sarcomas: A molecular analysis and review of the literature. Mutat. Res. 2010, 686, 68-73. [CrossRef] [PubMed]

63. Growdon, W.B.; Roussel, B.N.; Scialabba, V.L.; Foster, R.; Dias-Santagata, D.; Iafrate, A.J.; Ellisen, L.W.; Tambouret, R.H.; Rueda, B.R.; Borger, D.R. Tissue-specific signatures of activating PIK3CA and RAS mutations in carcinosarcomas of gynecologic origin. Gynecol. Oncol. 2011, 121, 212-217. [CrossRef] [PubMed]

64. Makker, V.; Recio, F.O.; Ma, L.; Matulonis, U.A.; Lauchle, J.O.; Parmar, H.; Gilbert, H.N.; Ware, J.A.; Zhu, R.; Lu, S.; et al. A multicenter, single-arm, open-label, phase 2 study of apitolisib (GDC-0980) for the treatment of recurrent or persistent endometrial carcinoma (MAGGIE study). Cancer 2016, 122, 3519-3528. [CrossRef] [PubMed]

65. López-García, M.Á.; Begoña, V.; Castilla, M.A.; Romero-Pérez, L.; Díaz-Martín, J.; Biscuola, M.; Palacios, C.J. Genetics of endometrial carcinoma. Cancer Genomics 2013, 11, 51.

66. Mills, A.M.; Liou, S.; Ford, J.M.; Berek, J.S.; Pai, R.K.; Longacre, T.A. Lynch syndrome screening should be considered for all patients with newly diagnosed endometrial cancer. Am. J. Surg. Pathol. 2014, 38, 1501-1509. [CrossRef]

67. Taylor, N.P.; Zighelboim, I.; Huettner, P.C.; Powell, M.A.; Gibb, R.K.; Rader, J.S.; Mutch, D.G.; Edmonston, T.B.; Goodfellow, P.J. DNA mismatch repair and TP53 defects are early events in uterine carcinosarcoma tumorigenesis. Mod. Pathol. 2006, 19, 1333-1338. [CrossRef] [PubMed]

68. Hoang, L.N.; Ali, R.H.; Lau, S.; Gilks, C.B.; Lee, C.H. Immunohistochemical survey of mismatch repair protein expression in uterine sarcomas and carcinosarcomas. Int. J. Gynecol. Pathol. 2014, 33, 483-491. [CrossRef]

69. Hembree, T.N.; Teer, J.K.; Hakam, A.; Chiappori, A.A. Genetic investigation of uterine carcinosarcoma: Case report and cohort analysis. Cancer Control 2016, 23, 61-66. [CrossRef]

70. Bhangoo, M.S.; Boasberg, P.; Mehta, P.; Elvin, J.A.; Ali, S.M.; Wu, W.; Klempner, S.J. Tumor mutational burden guides therapy in a treatment refractory POLE-mutant uterine carcinosarcoma. Oncologist 2018, 23, 518-523. [CrossRef]

71. Maxwell, G.L.; Chandramouli, G.V.; Dainty, L.; Litzi, T.J.; Berchuck, A.; Barrett, J.C.; Risinger, J.I. Microarray analysis of endometrial carcinomas and mixed mullerian tumors reveals distinct gene expression profiles associated with different histologic types of uterine cancer. Clin. Cancer Res. 2005, 11, 4056-4066. [CrossRef]

72. Whitehurst, A.W. Cause and consequence of cancer/testis antigen activation in cancer. Annu. Rev. Pharmacol. Toxicol. 2014, 54, 251-272. [CrossRef] [PubMed] 
73. Romero-Perez, L.; Castilla, M.A.; Lopez-Garcia, M.A.; Díaz-Martín, J.; Biscuola, M.; Ramiro-Fuentes, S.; Oliva, E.; Matias-Guiu, X.; Prat, J.; Cano, A.; et al. Molecular events in endometrial carcinosarcomas and the role of high mobility group AT-hook 2 in endometrial carcinogenesis. Hum. Pathol. 2013, 44, 244-254. [CrossRef] [PubMed]

74. Risinger, J.I.; Chandramouli, G.V.; Maxwell, G.L.; Custer, M.; Pack, S.; Loukinov, D.; Aprelikova, O.; Litzi, T.; Schrump, D.S.; Murphy, S.K.; et al. Global expression analysis of cancer/testis genes in uterine cancers reveals a high incidence of BORIS expression. Clin. Cancer Res. 2007, 13, 1713-1719. [CrossRef] [PubMed]

75. Resnick, M.B.; Sabo, E.; Kondratev, S.; Kerner, H.; Spagnoli, G.C.; Yakirevich, E. Cancer-testis antigen expression in uterine malignancies with an emphasis on carcinosarcomas and papillary serous carcinomas. Int. J. Cancer 2002, 101, 190-195. [CrossRef] [PubMed]

76. Roszik, J.; Wang, W.L.; Livingston, J.A.; Roland, C.L.; Ravi, V.; Yee, C.; Hwu, P.; Futreal, A.; Lazar, A.J.; Patel, S.R.; et al. Overexpressed PRAME is a potential immunotherapy target in sarcoma subtypes. Clin. Sarcoma Res. 2017, 7, 11. [CrossRef] [PubMed]

77. Ratner, E.S.; Tuck, D.; Richter, C.; Nallur, S.; Patel, R.M.; Schultz, V.; Hui, P.; Schwartz, P.E.; Rutherford, T.J.; Weidhaas, J.B. MicroRNA signatures differentiate uterine cancer tumor subtypes. Gynecol. Oncol. 2010, 118, 251-257. [CrossRef] [PubMed]

78. Gulyaeva, L.F.; Kushlinskiy, N.E. Regulatory mechanisms of microRNA expression. J. Transl. Med. 2016, 14, 143. [CrossRef] [PubMed]

79. Lei, Z.; Li, B.; Yang, Z.; Fang, H.; Zhang, G.M.; Feng, Z.H.; Huang, B. Regulation of HIF-1alpha and VEGF by miR-20b tunes tumor cells to adapt to the alteration of oxygen concentration. PLoS ONE 2009, 4, e7629. [CrossRef] [PubMed]

80. Hovey, A.M.; Devor, E.J.; Breheny, P.J.; Mott, S.L.; Dai, D.; Thiel, K.W.; Leslie, K.K. miR-888: A novel cancer-testis antigen that targets the progesterone receptor in endometrial cancer. Transl. Oncol. 2015, 8 , 85-96. [CrossRef]

81. Gonzalez Dos Anjos, L.; de Almeida, B.C.; Gomes de Almeida, T.; Mourão Lavorato Rocha, A.; De Nardo Maffazioli, G.; Soares, F.A.; Werneck da Cunha, I.; Chada Baracat, E.; Candido Carvalho, K. Could miRNA signatures be useful for predicting uterine sarcoma and carcinosarcoma prognosis and treatment? Cancers 2018, 10, 315. [CrossRef]

82. Li, J.; Xing, X.; Li, D.; Zhang, B.; Mutch, D.G.; Hagemann, I.S.; Wang, T. Whole-genome DNA methylation profiling identifies epigenetic signatures of uterine carcinosarcoma. Neoplasia 2017, 19, 100-111. [CrossRef] [PubMed]

83. Thiery, J.P.; Acloque, H.; Huang, R.Y.; Nieto, M.A. Epithelial-mesenchymal transitions in development and disease. Cell 2009, 139, 871-890. [CrossRef] [PubMed]

84. Diaz-Martin, J.; Diaz-Lopez, A.; Moreno-Bueno, G.; Castilla, M.Á.; Rosa-Rosa, J.M.; Cano, A.; Palacios, J. A core microRNA signature associated with inducers of the epithelial-to-mesenchymal transition. J. Pathol. 2014, 232, 319-329. [CrossRef] [PubMed]

85. Diaz-Lopez, A.; Diaz-Martin, J.; Moreno-Bueno, G.; Cuevas, E.P.; Santos, V.; Olmeda, D.; Portillo, F.; Palacios, J.; Cano, A. Zeb1 and Snail1 engage miR-200f transcriptional and epigenetic regulation during EMT. Int. J. Cancer 2015, 136, E62-E73. [CrossRef] [PubMed]

86. Berger, A.C.; Korkut, A.; Kanchi, R.S.; Hegde, A.M.; Lenoir, W.; Liu, W.; Liu, Y.; Fan, H.; Shen, H.; Ravikumar, V;; et al. A comprehensive pan-cancer molecular study of gynecologic and breast cancers. Cancer Cell 2018, 33, 690-705. [CrossRef] [PubMed]

87. Inoue, H.; Hashimura, M.; Akiya, M.; Chiba, R.; Saegusa, M. Functional role of ALK-related signal cascades on modulation of epithelial-mesenchymal transition and apoptosis in uterine carcinosarcoma. Mol. Cancer 2017, 16, 37. [CrossRef] [PubMed]

88. Chen, J.; Gao, F.; Liu, N. L1CAM promotes epithelial to mesenchymal transition and formation of cancer initiating cells in human endometrial cancer. Exp. Ther. Med. 2018, 15, 2792-2797. [CrossRef]

89. Versluis, M.; Plat, A.; de Bruyn, M.; Matias-Guiu, X.; Trovic, J.; Krakstad, C.; Nijman, H.W.; Bosse, T.; de Bock, G.H.; Hollema, H. L1CAM expression in uterine carcinosarcoma is limited to the epithelial component and may be involved in epithelial-mesenchymal transition. Virchows Arch. 2018, 473, 591-598. [CrossRef]

90. Jolly, M.K.; Jia, D.; Boareto, M.; Mani, S.A.; Pienta, K.J.; Ben-Jacob, E.; Levine, H. Coupling the modules of EMT and stemness: A tunable 'stemness window' model. Oncotarget 2015, 6, 25161-25174. [CrossRef] 
91. Pastushenko, I.; Blanpain, C. EMT transition states during tumor progression and metastasis. Trends Cell Biol. 2019, 29, 212-226. [CrossRef]

92. Wang, H.; Unternaehrer, J.J. Epithelial-mesenchymal transition and cancer stem cells: At the crossroads of differentiation and dedifferentiation. Dev. Dyn. 2019, 248, 10-20. [CrossRef] [PubMed]

93. Chal, J.; Pourquie, O. Making muscle: Skeletal myogenesis in vivo and in vitro. Development 2017, 144, 2104-2122. [CrossRef] [PubMed]

94. Lu, X.; Zhang, L.; Zhao, H.; Chen, C.; Wang, Y.; Liu, S.; Lin, X.; Wang, Y.; Zhang, Q.; Lu, T.; et al. Molecular classification and subtype-specific drug sensitivity research of uterine carcinosarcoma under multi-omics framework. Cancer Biol. Ther. 2019, 20, 227-235. [CrossRef] [PubMed]

95. Longoria, T.C.; Eskander, R.N. Immunotherapy in endometrial cancer-an evolving therapeutic paradigm. Gynecol. Oncol. Res. Pract. 2015, 2, 11. [CrossRef] [PubMed]

96. Ayers, M.; Lunceford, J.; Nebozhyn, M.; Murphy, E.; Loboda, A.; Kaufman, D.R.; Albright, A.; Cheng, J.D.; Kang, S.P.; Shankaran, V.; et al. IFN-gamma-related mRNA profile predicts clinical response to PD-1 blockade. J. Clin. Invest. 2017, 127, 2930-2940. [CrossRef] [PubMed]

97. Danaher, P.; Warren, S.; Lu, R.; Samayoa, J.; Sullivan, A.; Pekker, I.; Wallden, B.; Marincola, F.M.; Cesano, A. Pan-cancer adaptive immune resistance as defined by the Tumor Inflammation Signature (TIS): Results from The Cancer Genome Atlas (TCGA). J. Immunother. Cancer 2018, 6, 63. [CrossRef] [PubMed]

98. Dongre, A.; Rashidian, M.; Reinhardt, F.; Bagnato, A.; Keckesova, Z.; Ploegh, H.L.; Weinberg, R.A. Epithelial-to-mesenchymal transition contributes to immunosuppression in breast carcinomas. Cancer Res. 2017, 77, 3982-3989. [CrossRef]

99. Ueno, T.; Tsuchikawa, T.; Hatanaka, K.C.; Hatanaka, Y.; Mitsuhashi, T.; Nakanishi, Y.; Noji, T.; Nakamura, T.; Okamura, K.; Matsuno, Y.; et al. Prognostic impact of programmed cell death ligand 1 (PD-L1) expression and its association with epithelial-mesenchymal transition in extrahepatic cholangiocarcinoma. Oncotarget 2018, 9, 20034-20047. [CrossRef] [PubMed]

100. Noman, M.Z.; Janji, B.; Abdou, A.; Hasmim, M.; Terry, S.; Tan, T.Z.; Mami-Chouaib, F.; Thiery, J.P.; Chouaib, S. The immune checkpoint ligand PD-L1 is upregulated in EMT-activated human breast cancer cells by a mechanism involving ZEB-1 and miR-200. Oncoimmunology 2017, 6, e1263412. [CrossRef]

101. Kim, S.; Koh, J.; Kim, M.Y.; Kwon, D.; Go, H.; Kim, Y.A.; Jeon, Y.K.; Chung, D.H. PD-L1 expression is associated with epithelial-to-mesenchymal transition in adenocarcinoma of the lung. Hum. Pathol. 2016, 58, 7-14. [CrossRef]

102. Ock, C.Y.; Kim, S.; Keam, B.; Kim, M.; Kim, T.M.; Kim, J.H.; Jeon, Y.K.; Lee, J.S.; Kwon, S.K.; Hah, J.H.; et al. PD-L1 expression is associated with epithelial-mesenchymal transition in head and neck squamous cell carcinoma. Oncotarget 2016, 7, 15901-15914. [CrossRef] [PubMed]

103. Mak, M.P.; Tong, P.; Diao, L.; Cardnell, R.J.; Gibbons, D.L.; William, W.N.; Skoulidis, F.; Parra, E.R.; Rodriguez-Canales, J.; Wistuba, I.I.; et al. A patient-derived, pan-cancer EMT signature identifies global Molecular alterations and immune target enrichment following epithelial-to-mesenchymal transition. Clin. Cancer Res. 2016, 22, 609-620. [CrossRef] [PubMed]

104. Chen, L.; Gibbons, D.L.; Goswami, S.; Cortez, M.A.; Ahn, Y.H.; Byers, L.A.; Zhang, X.; Yi, X.; Dwyer, D.; Lin, W.; et al. Metastasis is regulated via microRNA-200/ZEB1 axis control of tumour cell PD-L1 expression and intratumoral immunosuppression. Nat. Commun. 2014, 5, 5241. [CrossRef] [PubMed]

105. Dong, P.; Xiong, Y.; Yue, J.; Hanley, S.J.B.; Watari, H. Tumor-intrinsic PD-L1 signaling in cancer initiation, development and treatment: Beyond immune evasion. Front. Oncol. 2018, 8, 386. [CrossRef] [PubMed]

106. Velcheti, V.; Rimm, D.L.; Schalper, K.A. Sarcomatoid lung carcinomas show high levels of programmed death ligand-1 (PD-L1). J. Thorac. Oncol. 2013, 8, 803-805. [CrossRef] [PubMed]

107. Zhang, Z.; Chen, Y.; Ma, M.; Hao, J.; Ding, R.; Han, L.; Zou, J.; Zhang, L.; Meng, Q.; Qu, X.; et al. Significant benefit of Nivolumab treating PD-L1 positive metastatic pulmonary carcinosarcoma: A case report and literature review. Oncotarget 2017, 8, 96453-96459. [CrossRef]

108. Zhu, J.; Wen, H.; Ju, X.; Bi, R.; Zuo, W.; Wu, X. Clinical significance of programmed death ligand1 and intra-tumoral CD8+ T lymphocytes in ovarian carcinosarcoma. PLoS ONE 2017, 12, e0170879.

109. Pinto, A.; Mackrides, N.; Nadji, M. PD-L1 expression in carcinosarcomas of the gynecologic tract: A potentially actionable biomarker. Appl. Immunohistochem. Mol. Morphol. 2018, 26, 393-397. [CrossRef]

110. Remmerie, M.; Janssens, V. Targeted therapies in type II endometrial cancers: Too little, but not too late. Int. J. Mol. Sci. 2018, 19, 2380. [CrossRef] 
111. Clinical Trials. Available online: clinicaltrials.gov (accessed on 26 June 2019).

112. Fader, A.N.; Roque, D.M.; Siegel, E.; Buza, N.; Hui, P.; Abdelghany, O.; Chambers, S.K.; Secord, A.A.; Havrilesky, L.; O'Malley, D.M.; et al. Randomized phase II trial of carboplatin-paclitaxel versus carboplatin-paclitaxel-trastuzumab in uterine serous carcinomas that overexpress human epidermal growth factor receptor 2/neu. J. Clin. Oncol. 2018, 36, 2044-2051. [CrossRef]

(C) 2019 by the authors. Licensee MDPI, Basel, Switzerland. This article is an open access article distributed under the terms and conditions of the Creative Commons Attribution (CC BY) license (http://creativecommons.org/licenses/by/4.0/). 